\title{
A Novel Mutation of ORNT1 Detected in a Hyperornithinemia-Hyperammonemia- Homocitrullinuria Syndrome Child by Clinical Whole-Exome Sequencing
}

\author{
Wison Laochareonsuk ${ }^{1}$ Seksit Osatakul ${ }^{2} \quad$ Utcharee Intusoma $^{3} \quad$ Wanwisa Maneechay ${ }^{4}$ \\ Surasak Sangkhathat ${ }^{4,5}$
}

${ }^{1}$ Division of Biomedical Science and Biomedical Engineering, Faculty of Medicine, Prince of Songkla University, Songkhla, Thailand

2 Pediatric Gastroenterology Unit, Division of Pediatrics, Faculty of Medicine, Prince of Songkla University, Songkhla, Thailand

3 Pediatric Neurology Unit, Division of Pediatrics, Faculty of Medicine, Prince of Songkla University, Songkhla, Thailand

${ }^{4}$ Translational Medicine Research Center, Faculty of Medicine, Prince of Songkla University, Songkhla, Thailand

${ }^{5}$ Division of Surgery, Faculty of Medicine, Prince of Songkla

University, Songkhla, Thailand

Address for correspondence Surasak Sangkhathat, MD, PhD, Division of Surgery, Translational Medicine Research Center, Faculty of Medicine, Prince of Songkla University, Hat Yai, Songkhla 90110, Thailand (e-mail: surasak.sa@psu.ac.th).

J Pediatr Genet

\begin{abstract}
Keywords

- SLC25A15 gene

- whole-exome sequencing

- urea cycle disorders

Hyperornithinemia-hyperammonemia-homocitrullinuria (HHH) syndrome, an inborn error of metabolism, is an inherited syndrome caused by loss-of-function mutations in the SLC25A15, resulting in ornithine translocase1 (ORNT1) deficiency. Disrupted ornithine transportation in an affected individual usually manifests with the accumulation of intermediate metabolites, leading to neurological impairment, hepatitis, and/or protein intolerance at various ages of onset. In this paper, we report a compound heterozygous mutation in SLC25A15 from a 2-year-old girl who presented with neurological alterations and hepatic failure. Before developing neurological sequelae, she had signs of globally delayed development. The accumulation of toxic metabolites may explain these neurological consequences. After biochemical confirmation of $\mathrm{HHH}$, whole-exome sequencing (WES) was performed, which identified mutations at codons 21 and 179 of SLC25A15 that are predicted to result in the loss of function of ORNT1. Each of the mutations was found to be inherited from one of her parents. After therapy, her toxic metabolites decreased significantly. In conclusion, $\mathrm{HHH}$ syndrome frequently manifests with nonspecific symptoms and unapparent biochemical profiles, which may lead to delayed diagnosis. Correction of the accumulating metabolites is necessary to prevent irreversible neurological impairment. Furthermore, performing a WES provides a shortcut for accurate diagnosis.
\end{abstract}

received

August 24, 2021

accepted after revision

December 21, 2021
DOI https://doi.org/

$10.1055 / \mathrm{s}-0041-1742247$.

ISSN 2146-4596.

\footnotetext{
(C) 2022. The Author(s).

This is an open access article published by Thieme under the terms of the Creative Commons Attribution-NonDerivative-NonCommercial-License, permitting copying and reproduction so long as the original work is given appropriate credit. Contents may not be used for commercial purposes, or adapted, remixed, transformed or built upon. (https://creativecommons.org/ licenses/by-nc-nd/4.0/) Georg Thieme Verlag KG, Rüdigerstraße 14, 70469 Stuttgart, Germany
} 


\section{Introduction}

Hyperornithinemia-hyperammonemia-homocitrullinuria (HHH) syndrome is a rare hereditary urea cycle disorder (UCD) found in approximately 1:2,000,000 livebirths. ${ }^{1}$ The syndrome is caused by an ornithine translocase1 (ORNT1) deficiency secondary to homozygous or compound heterozygous mutations that result in the loss of function of the SLC25A15 gene. ${ }^{2}$ Ornithine is an intermediate metabolite in the urea cycle which transfers carbamoyl phosphate from the mitochondrial matrix to the cytosol. Defects of ORNT1 disrupt ornithine and citrulline transportation, leading to the accumulation of the precursor substrates in the plasma, as well as ornithine and ammonia, and retained carbamoyl phosphate is alternatively converted to homocitrulline and excreted through the urine. ${ }^{3}$

As a result of episodic increases of plasma ammonia, an affected individual can present at various ages of onset with a wide spectrum of manifestations including protein-rich diet intolerance, neurological impairment, and hepatitis. ${ }^{4,5}$ To prevent sequelae of ammonia toxicity, acute treatment is required, especially prompt reduction of plasma ammonia and correction of metabolite imbalances. For maintenance therapy, recommendations include a low-protein diet combined with essential amino acid supplements such as citrulline or arginine to archive biochemical control. In addition, some patients will need ammonia scavengers including sodium benzoate or sodium phenylbutyrate to maintain appropriate serum ammonia. ${ }^{6}$ Long-term neurological outcomes vary from mild intellectual disturbances to severe disability.

Because of rapidly decreasing costs and increasing accuracy of high throughput technology, clinical whole-exome sequencing (WES) can potentially help clinicians to provide a timely diagnosis and appropriate treatment for rare pediatric diseases. Early diagnosis of an inborn error of metabolism leads to prompt implementation of appropriate metabolic control which improves long-term clinical outcomes.
In this case report, we described a girl with $\mathrm{HHH}$ syndrome who presented with neurological alterations and hepatic failure at the age of 2 years. Clinical WES comprehensively annotated the whole panel of genes involved in her UCDs. Validated data from the WES were then used to narrow down the involved metabolites. Consistency between the genotype and biochemical profile led to the confirmation of the diagnosis.

\section{Case Presentation}

The proband was a 2-year-old girl child patient born following an uncomplicated G1P0 pregnancy by spontaneous vaginal delivery without remarkable obstetric complications. Her family had no known genetic disorders. The infant was thought to be in good health until she was identified with globally delayed development on her first visit to a well-child developmental screening clinic. At the age of 12 months, she was not able to sit without support and she struggled with grasping and releasing objects and could not pronounce even single-syllable words. Her parents had also noticed that she usually refused to eat pork or chicken. Before she was admitted, the patient had consulted a pediatrician at a provincial hospital about vomiting and acute alteration of consciousness, beginning 2 days prior to that visit. The local hospital found that the patient had metabolic acidosis and treated her with intravenous fluid before referral to our institute. At the admission, a physical examination revealed gross lethargy, hypotonia, and hyperreflexia of all extremities without motor power or cranial nerve defects. Her biochemical tests including serum electrolytes, blood urea nitrogen, and creatinine were normal, but the liver function test showed transaminitis and coagulopathy (-Table $\mathbf{1}$ ). Initially, acute hepatic encephalopathy was diagnosed, and she was treated with oral lactulose solution and a vitamin $\mathrm{K}$ injection to desensitize the hepatocellular toxicity and correct the coagulopathy.

The pediatrician decided to additionally screen the child for other potential causes of hepatitis including

Table 1 Liver function test and coagulogram of the patient at the day of diagnosis and consequent admissions

\begin{tabular}{|l|l|l|l|l|l|}
\hline Profiles & Before treatment & Before referral & After referral & After treatment & Reference \\
\hline Directed bilirubin & 0.34 & 0.26 & 0.31 & 0.40 & $0-0.3 \mathrm{mg} \%$ \\
\hline Total bilirubin & 0.4 & 0.53 & 0.55 & 1.10 & $0-1.2 \mathrm{mg} \%$ \\
\hline Alanine aminotransferase & 176 & 177 & 1,017 & 144 & $\leq 66$ \\
\hline Aspartate aminotransferase & 574 & 484 & 2,025 & $266 / \mathrm{L}$ \\
\hline Alkaline phosphatase & 202 & 188 & 313 & 284 & $<\leq 33 \mathrm{U} / \mathrm{L}$ \\
\hline Total protein & 7.0 & 6.8 & 6.9 & 6.6 & $<291 \mathrm{U} / \mathrm{L}$ \\
\hline Albumin & 4.3 & 4.1 & 4.4 & 4.2 & $6.4-8.3 \mathrm{~g} \%$ \\
\hline Partial thromboplastin time (PTT) & 40.5 & 49.6 & 60.5 & 47.0 & $3.5-5.2 \mathrm{~g} \%$ \\
\hline Prothrombin time (PT) & 37.2 & 28.8 & 38.7 & 24.4 & $21.0-30.3 \mathrm{~s}$ \\
\hline PTT ratio & 1.62 & 1.99 & 2.35 & 1.83 & $10.1-12.1 \mathrm{~s}$ \\
\hline PT international normalized ratio & 3.69 & 2.67 & 3.81 & 2.32 & 9.7 \\
\hline Thrombin time & & 14.3 & & & $8.4-11.1 \mathrm{~s}$ \\
\hline
\end{tabular}


infectious hepatitis and autoimmune hepatitis. However, all studies were negative. After 3 days of treatment, her general status improved, and she regained consciousness. However, her liver functions and coagulation status remained unstable. She was then transferred to the pediatric gastroenterology unit of our institute for further investigations to explore possible metabolic causes of her condition. Her serum ammonia was significantly elevated at $124 \mathrm{mcg} / \mathrm{dL}$, but with normal levels of serum ceruloplasmin and anti-nuclear antibodies were regular (-Table 2). Then, a peripheral blood sample was taken for a quantitative amino acid analysis and WES. She was put on a strict diet in which protein was restricted to below $1 \mathrm{~g} / \mathrm{kg} /$ day and measures were taken to correct her metabolic imbalances. Within 48 hours, she regained full consciousness without seizures or confusion and over the next few days her biochemistry gradually improved. She was discharged 10 days after admission, when her coagulation was fully normalized. When the WES results were analyzed, the variants prioritization suggested that the child's symptoms could be explained by a diagnosis of ORNT1 deficiency. Plasma amino acid analysis (Mayo Clinic Laboratory, United States) showed serum ornithine at a very high level at $337 \mathrm{nmol} / \mathrm{dL}$ ( - Table 3), together with urine homocitrulline at $241 \mathrm{nmol} / \mathrm{mg}$ creatinine. These findings led to a definite diagnosis of $\mathrm{HHH}$ syndrome. The patient was then supplemented with L-citrulline and sodium
Table 2 Electrolyte and specific biochemistry of the patient at the time of admission

\begin{tabular}{|l|l|l|}
\hline Chemistry & Value & Reference \\
\hline Blood urea nitrogen & $12.9 \mathrm{mg} \%$ & $6-20 \mathrm{mg} \%$ \\
\hline Creatinine & $0.13 \mathrm{mg} \%$ & - \\
\hline Sodium & $138.0 \mathrm{mmol} / \mathrm{L}$ & $136-145 \mathrm{mmol} / \mathrm{L}$ \\
\hline Potassium & $4.47 \mathrm{mmol} / \mathrm{L}$ & $3.4-4.5 \mathrm{mmol} / \mathrm{L}$ \\
\hline Chloride & $105.6 \mathrm{mmol} / \mathrm{L}$ & $98-107 \mathrm{mmol} / \mathrm{L}$ \\
\hline Bicarbonate & $18.8 \mathrm{mmol} / \mathrm{L}$ & $22-29 \mathrm{mmol} / \mathrm{L}$ \\
\hline Calcium & $9.8 \mathrm{mg} \%$ & $8.6-10.2 \mathrm{mg} \%$ \\
\hline Phosphorus & $4.2 \mathrm{mg} \%$ & $4-7 \mathrm{mg} \%$ \\
\hline Serum ceruloplasmin & $21 \mathrm{mg} \%$ & \\
\hline Serum ammonia & $124.0 \mathrm{mcg} / \mathrm{dL}$ & $12-66 \mathrm{mcg} / \mathrm{dL}$ \\
\hline Urine copper & $0.031 \mathrm{mg} / \mathrm{L}$ & \\
\hline
\end{tabular}

benzoate at a starting dosage of $250 \mathrm{mg} / \mathrm{kg} /$ day, following which her serum ammonia decreased significantly over 3 weeks. At her latest follow-up at the age of 3 years, her serum ammonia was still within normal limits (45 $\mathrm{mcg} / \mathrm{dL}$ ), could walk without assistance, and was able to formulate long sentences and communicate with other children.

Table 3 Serum quantitative amino acid validation of the patient

\begin{tabular}{|c|c|c|c|c|c|}
\hline Amino acid & Value & Reference & Amino acid & Value & Reference \\
\hline Phosphoserine & 0 & $<95 \mathrm{nmol} / \mathrm{mL}$ & Histidine & 128 & $12-132 \mathrm{nmol} / \mathrm{mL}$ \\
\hline Phosphoethanolamine & 3 & $<5 \mathrm{nmol} / \mathrm{mL}$ & Threonine & 84 & $58-195 \mathrm{nmol} / \mathrm{mL}$ \\
\hline Taurine & 81 & $38-153 \mathrm{nmol} / \mathrm{mL}$ & Citrulline & 31 & $11-45 \mathrm{nmol} / \mathrm{mL}$ \\
\hline Asparagine & 86 & $29-87 \mathrm{nmol} / \mathrm{mL}$ & Sarcosine & 5 & $<5 \mathrm{nmol} / \mathrm{mL}$ \\
\hline Serine & 152 & $71-208 \mathrm{nmol} / \mathrm{mL}$ & Beta-alanine & 15 & $<27 \mathrm{nmol} / \mathrm{mL}$ \\
\hline Hydroxyproline & 15 & $7-35 \mathrm{nmol} / \mathrm{mL}$ & Alanine & 731 & $144-557 \mathrm{nmol} / \mathrm{mL}$ \\
\hline Glycine & 260 & $149-417 \mathrm{nmol} / \mathrm{mL}$ & Glutamic acid & 39 & $22-131 \mathrm{nmol} / \mathrm{mL}$ \\
\hline Glutamine & 1,510 & $239-976 \mathrm{nmol} / \mathrm{mL}$ & 1-Methylhistidine & 0 & $<20 \mathrm{nmol} / \mathrm{mL}$ \\
\hline Aspartic acid & 3 & $<11 \mathrm{nmol} / \mathrm{mL}$ & 3-Methylhistidine & 1 & $<1 \mathrm{nmol} / \mathrm{mL}$ \\
\hline Ethanolamine & $<7$ & $<64 \mathrm{nmol} / \mathrm{mL}$ & Proline & 254 & $80-357 \mathrm{nmol} / \mathrm{mL}$ \\
\hline Argininosuccinic acid & 0 & $<2 \mathrm{nmol} / \mathrm{mL}$ & Ornithine & 377 & $22-97 \mathrm{nmol} / \mathrm{mL}$ \\
\hline Carnosine & 2 & $<1 \mathrm{nmol} / \mathrm{mL}$ & Cystathionine & $<1$ & $<2 \mathrm{nmol} / \mathrm{mL}$ \\
\hline Anserine & 0 & $<1 \mathrm{nmol} / \mathrm{mL}$ & Cystine & 37 & $2-36 \mathrm{nmol} / \mathrm{mL}$ \\
\hline Homocitrulline & 2 & $<2 \mathrm{nmol} / \mathrm{mL}$ & Lysine & 135 & $59-240 \mathrm{nmol} / \mathrm{mL}$ \\
\hline Arginine & 49 & $31-132 \mathrm{nmol} / \mathrm{mL}$ & Methionine & 61 & $11-37 \mathrm{nmol} / \mathrm{mL}$ \\
\hline Alpha-aminoadipic acid & 2 & $<3 \mathrm{nmol} / \mathrm{mL}$ & Valine & 163 & $106-320 \mathrm{nmol} / \mathrm{mL}$ \\
\hline Gamma-amino-n-butyric acid & 0 & $<3 \mathrm{nmol} / \mathrm{mL}$ & Tyrosine & 88 & $31-106 \mathrm{nmol} / \mathrm{mL}$ \\
\hline Beta-aminoisobutyric acid & 2 & $<5 \mathrm{nmol} / \mathrm{mL}$ & Isoleucine & 66 & $30-111 \mathrm{nmol} / \mathrm{mL}$ \\
\hline Alpha-amino-n-butyric acid & 33 & $7-31 \mathrm{nmol} / \mathrm{mL}$ & Leucine & 103 & $51-196 \mathrm{nmol} / \mathrm{mL}$ \\
\hline Hydroxylysine & 0 & $<3 \mathrm{nmol} / \mathrm{mL}$ & Phenylalanine & 61 & $30-95 \mathrm{nmol} / \mathrm{mL}$ \\
\hline Tryptophan & 64 & $23-80 \mathrm{nmol} / \mathrm{mL}$ & Allo-isoleucine & 0 & $<3 \mathrm{nmol} / \mathrm{mL}$ \\
\hline
\end{tabular}




\section{Molecular Genetic Study}

The peripheral blood sample was obtained from the patient and all family members with an informed consent. Genomic DNA was then extracted from the blood leukocytes, and the quality and quantity were checked. The DNA of the proband underwent exome capture and adapter ligation using Agilent SureSelect XT. Massive parallel sequencing was performed on paired-end reads using an Illumina platform. The quality of sequencing data was verified and found to have an average base quality score at Q30 more than 90\% of the overall sequences. Using bioinformatic tools, the sequence reads were computed using Burrows-Wheeler Aligner and a Genome Analysis Toolkit pipeline with reference-based alignment. On mapping the sequences to the latest human genome reference (GRCh38.p13), more than $90 \%$ of the reads covered $40 \mathrm{X}$ of exome regions. During the discovery process, the identified variants were annotated with several clinical databases, namely the Human Gene Mutation Database, the non-synonymous single-nucleotide variants database, and the Genome Aggregation Database (-Supplementary Figure S1, available in online version only). The possible variants were filtered based on the criteria of allele calling at more than $25 \%$ of supporting reads at the depth of coverage of more than 40x and minor allele frequencies less than 0.01 in East Asian populations. Priority was given to rare homozygous variants and compound heterozygous variants occurring to genes in the urea cycle pathway (-Supplementary Figure S2, available in online version only).

After prioritization, the most likely causative mutations were found to be located in exons 3 and 5 of SLC25A15 at positions g.40799063C $>$ A and g.40807376C $>$ T of chromosome 13. Those variants caused a missense mutation of codon 21 (p.Thr21Lys) and a nonsense mutation of codon 179 (p.Arg179Ter). A mutation plot of the identified pathogenic variants in SLC25A15 gene was made using the trackViewer package of R software (-Fig. 1). ${ }^{7}$ The novel missense mutation was validated by an in silico prediction database using PROVEAN and SIFT. The reports showed deleterious $(-3.22)$ in PROVEAN and damaging $(0.001)$ in SIFT. Those variants were then assessed in the girl's parents using polymerase chain reaction and Sanger sequencing. The results evidenced a pattern of autosomal recessive inheritance of ORNT1, in which variant at position 40807376 was inherited from the mother and at position 40799063 was from the father (-Fig. 2 ).

\section{Discussion}

The urea cycle, an essential pathway in ammonia metabolism, requires sequential functions of six catalytic enzymes and two transportation processes in both the cytoplasm and mitochondrial matrix. Defects of urea synthesis lead to inherited inborn errors of metabolism. Affected patients are commonly presented with hyperammonemia and accumulation of intermediate metabolites. Most of UCDs are caused by autosomal recessive inheritance that may manifest with various spectra of onset and severity. ${ }^{8}$ Typically, acute hyperammonemia is an emergency condition. If not properly treated, an affected individual will potentially succumb to the disease. To maintain metabolites within normal ranges in affected people, a low-protein diet and essential amino acid supplements are provided. The aims of long-term follow-ups include reducing the effects of permanent neurocognitive disabilities and preventing intermittent hyperammonemia crises. ${ }^{9,10}$ The incidence of ORNT1 deficiency caused by lossof-function mutations of the SLC25A15 is estimated at $1: 2,000,000$ livebirths and is most common in Canada, France, Japan, and the Middle East. ${ }^{3}$ SLC25A15, encodes ORNT1 that contains 310 amino acids, which is located on chromosome 13q14.11 and contains 7 exons. An ORNT1 deficiency leads to the loss of transportation of ornithine, which is required for citrulline assembly. Normally, individuals with definitive metabolite disturbances of $\mathrm{HHH}$ syndrome manifesting as ORNT1 deficiency have the triad of symptoms of HHH. Diagnosis of HHH syndrome is frequently delayed or misdiagnosed from nonspecific presenting symptoms and insufficient biochemical studies (-Fig. 3A). A clinical WES study directly examines specific genes involved in a specific metabolic pathway and can lead to rapid

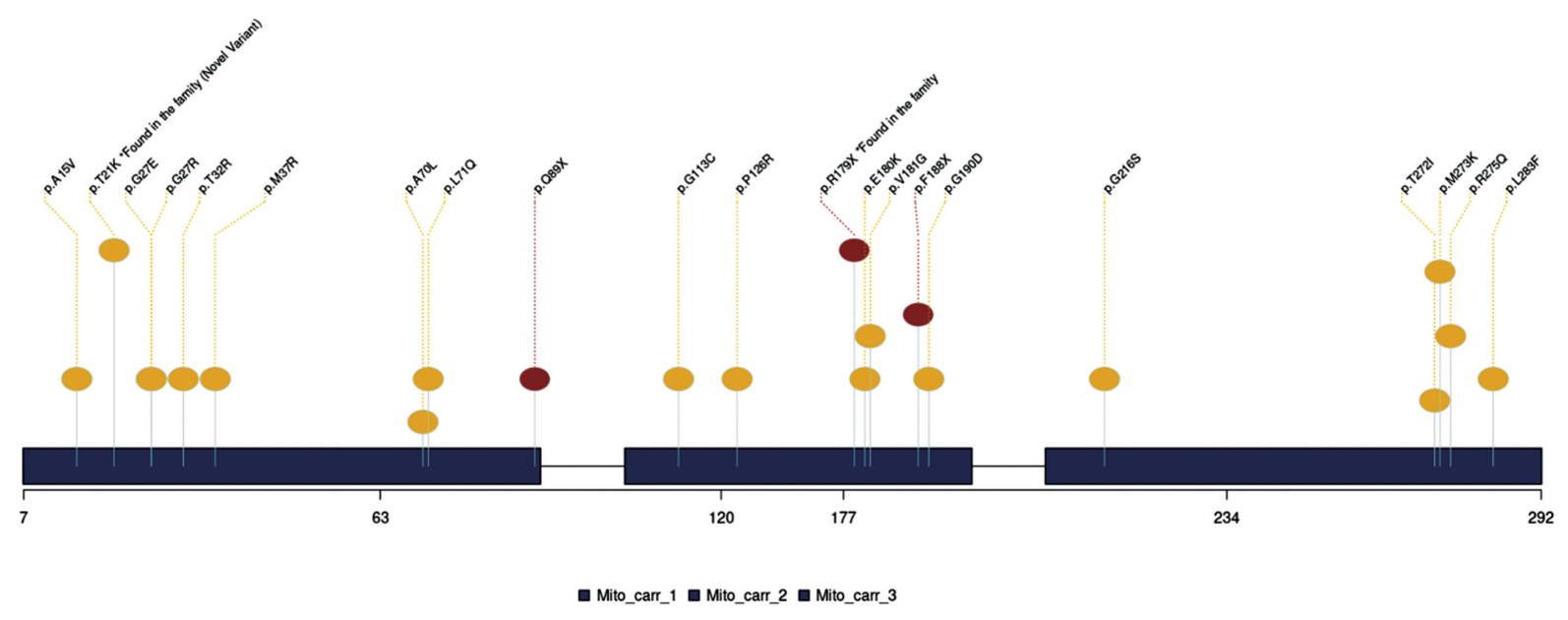

Fig. 1 Mutation plot of pathogenic variants of SLC25A15 identified in our family and earlier reports. 


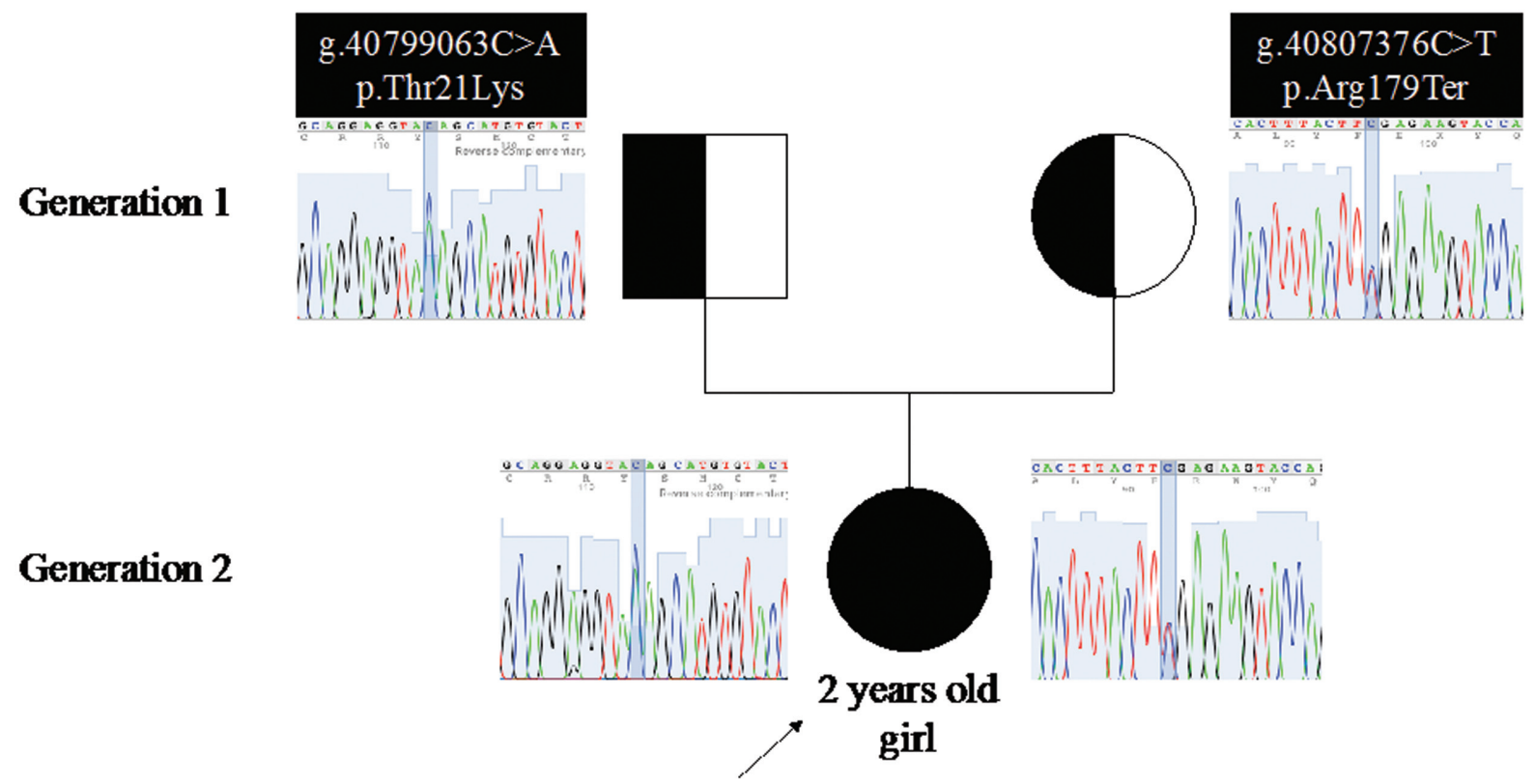

Fig. 2 The pedigree shows the inheritant pattern of compound heterozygous mutations of SLC25A15 at codon 21 (p.Thr21 Lys) and codon 179 (p.Arg179Ter).

(a)

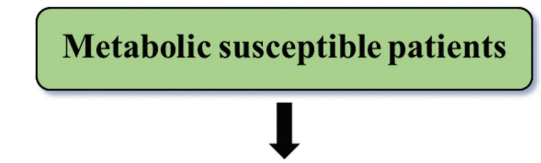

Metabolic screening test

Elevated serum ammonia

$\begin{gathered}\text { Suspicious of urea cycle } \\ \text { disorders }\end{gathered}$
.
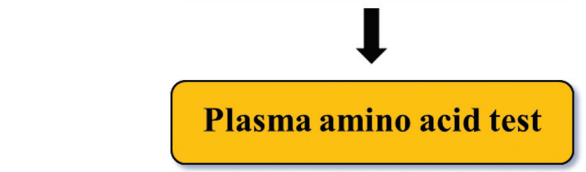

Mislead by insufficient method

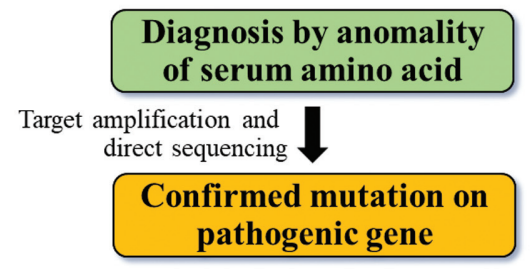

(b)

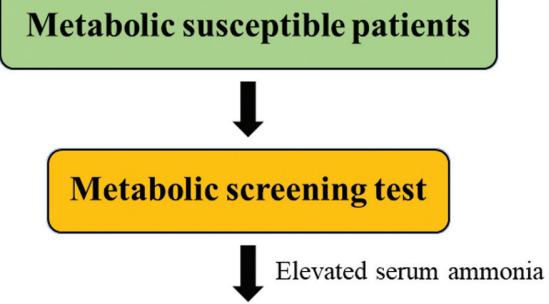

Suspicious of urea cycle disorders

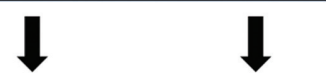

Plasma amino acid test

Genomic Sequencing

Dual high-throughput techniques



Diagnosis by both of results from proteomic and genomic studies

Fig. 3 The comparison of diagnosis schema between (A) traditional algorithm and (B) purposed algorithm.

diagnosis of HHH syndrome and other UCDs. In our case, the diagnosis of HHH syndrome was confirmed by conventional molecular genetic analysis in both the patient as well as her family and also by amino acid quantification (-Fig. 3B). The trio study confirmed that the patient carried compound heterozygous mutations in SLC25A15 of codon 21 in combination with codon 179. p.Arg179Ter, which is a well-known mutation found in 25 to $30 \%$ of HHH patients. ${ }^{11}$ The hetero- zygosity status of p.Arg179Ter with the biochemistry profile showing severe hyperornithinemia and the mutation $\mathrm{p}$. Thr21Lys present in the other allele, which is likely to cause loss-of-function of ORNT1 in a compound heterozygous mode of inheritance. As p.Thr21Lys has not been reported before, the novel mutation in this case could serve as an evidence for variant annotation in an infant suspected of $\mathrm{HHH}$. Thr21 is located at the inner mitochondrial membrane 
portion of the protein, which could be functionally involved in transporting ornithine, lysine, and arginine in exchange for citrulline. The majority of SLC25A15 mutations reported in $\mathrm{HHH}$ patients are more commonly recorded in this part of ORNT1. ${ }^{2}$

Looking back to the first visit, the child was originally brought to the hospital due to alteration of consciousness and vomiting, following which hyperammonemia, transaminitis, and coagulopathy were detected. Although her global development delay might have suggested metabolic disorders, her physical examination showed an indistinct, non-specific neurological deficit. As a high-resolution serum protein electrophoresis was not available in our setting, clinical WES became a valuable tool in approaching the hyperammonemia-related conditions in this patient. A genomic approach is particularly valuable when there are fluctuations of serum chemistry in patients who have received partial treatment. In $\mathrm{HHH}$, abnormalities in serum amino acids vary with types and amounts of protein intake. In our patient, the serum homocitrulline was borderline, but this was inconclusive as the blood sample was taken after the acute life-threatening symptoms stage had passed. Mutation data derived from WES were an adjunct to the biochemical studies in the confirmation of the diagnosis. After ruling out various causes of hepatic failure, aggressive elimination of ammonia and protein restriction was started. To rapidly reach an accurate diagnosis in this urgent situation, we have performed genetic and proteomic studies to delineate the cause of this disorder. When the definite diagnosis was made, she received additional amino acid supplements and ammonia scavenger drugs. Ultimately, her clinical and metabolic problems were successfully resolved.

\section{Conclusion}

In conclusion, $\mathrm{HHH}$ syndrome is a rare congenital disorder found in a very wide range of differing populations. Due to a variety of presentations and frequently misrecognized biochemical profiles, affected individuals usually have delayed or misdiagnoses, often leading to neurological disabilities. In recent years, WES has rapidly decreased in cost and has increased accuracy that enables clinicians to gain the information of causative mutations that can be interpreted together with the standard quantification of aberrant metabolites. In $\mathrm{HHH}$ cases, early diagnosis is essential for both emergency correction of the metabolites and beginning for the required managing therapies with supplementations.

\section{Funding}

None.

\section{Conflict of Interest}

None declared.

\section{Acknowledgment}

The authors would like to thank Dave Patterson of the Office of International Affairs, Faculty of Medicine, Prince of Songkla University for editing the English language in the manuscript.

\section{References}

1 Summar ML, Koelker S, Freedenberg D, et al; European Registry and Network for Intoxication Type Metabolic Diseases (E-IMD). Electronic address: http://www.e-imd.org/en/index.phtml Members of the Urea Cycle Disorders Consortium (UCDC). Electronic address: http://rarediseasesnetwork.epi.usf.edu/ucdc/. The incidence of urea cycle disorders. Mol Genet Metab 2013;110(1-2):179-180

2 Camacho JA, Obie C, Biery B, et al. Hyperornithinaemia-hyperammonaemia-homocitrullinuria syndrome is caused by mutations in a gene encoding a mitochondrial ornithine transporter. Nat Genet 1999;22(02):151-158

3 Martinelli D, Diodato D, Ponzi E, et al. The hyperornithinemiahyperammonemia-homocitrullinuria syndrome. Orphanet J Rare Dis 2015;10(01):29

4 Debray F-G, Lambert M, Lemieux B, et al. Phenotypic variability among patients with hyperornithinaemia-hyperammonaemiahomocitrullinuria syndrome homozygous for the delF188 mutation in SLC25A15. J Med Genet 2008;45(11):759-764

5 Salvi S, Santorelli FM, Bertini E, et al. Clinical and molecular findings in hyperornithinemia-hyperammonemia-homocitrullinuria syndrome. Neurology 2001;57(05):911-914

6 Häberle J, Boddaert N, Burlina A, et al. Suggested guidelines for the diagnosis and management of urea cycle disorders. Orphanet J Rare Dis 2012;7(01):32

7 Ou J, Zhu LJ. trackViewer: a bioconductor package for interactive and integrative visualization of multi-omics data. Nat Methods 2019;16(06):453-454

8 Matsumoto S, Häberle J, Kido J, Mitsubuchi H, Endo F, Nakamura K. Urea cycle disorders-update. J Hum Genet 2019;64(09):833-847

9 Batshaw ML, Tuchman M, Summar M, Seminara JMembers of the Urea Cycle Disorders Consortium. A longitudinal study of urea cycle disorders. Mol Genet Metab 2014;113(1-2):127-130

10 Ho B, MacKenzie J, Walia J, et al. Hyperornithinemia-hyperammonemia-homocitrullinuria syndrome in pregnancy: considerations for management and review of the literature. JIMD Rep 2019;46(01):28-34

11 Tessa A, Fiermonte G, Dionisi-Vici C, et al. Identification of novel mutations in the SLC25A15 gene in hyperornithinemia-hyperammonemia-homocitrullinuria ( $\mathrm{HHH})$ syndrome: a clinical, molecular, and functional study. Hum Mutat 2009;30(05):741-748 